\title{
Fragen zum Ort von Gesinnungskräften im publizistischen Funktionieren
}

\author{
von Michael Schmolke
}

Die alte Zeitungswissenschaft, wenngleich der Name noch nicht sterben will, ist tot, gehört der Wissenschaftsgeschichte an. Selbst die von Emil Dovifat und Walter Hagemann geprägte Publizistikwissenschaft hat sich - in ihrer Urform - als ein Durchgangsstadium erwiesen. Allenthalben an deutschen Fachinstituten, - denn es handelte sich bei der Zeitungswissenschaft um eine vorwiegend deutsch-mitteleuropäisch geprägte Schule, - sucht man seit einigen Jahren nach neuen Orientierungen, Methoden, Gegenständen und schließlich auch nach einer neuen publizistischen Philosophie. Auf diesen Ubergang, der die Phase des Tastens und ersten Versuchens schon hinter sich gelassen hat, muß im Rahmen der "Communicatio socialis" nicht zuletzt deshalb hingewiesen werden, weil nicht wenige der führenden Köpfe der Zeitungsund frühen Publizistikwissenschaft katholischer Konfession und einige sogar ausgesprochen gesellschaftlich aktive Katholiken waren. Um nur die bekanntesten Namen zu nennen: Karl d'Ester, Walter Hagemann und Emil Dovifat, der heute noch als Anreger der Jungen die Tradition mit dem Neuen verknüpfen hilft, gehören zu ihnen, - aber auch Karl Bömer, bis heute nicht ersetzter Bibliograph der Zeitungswissenschaft ${ }^{1}$, und der fast vergessene, in seinen frühen wissenschaftlichen Leistungen jedoch herausragende und wegweisende Josef Hardy Krumbach ${ }^{2}$.

Kennzeichnend für diesen „zweiten Ansatz" der Zeitungswissenschaft, den man vom ersten, etwas stärker rational-deskriptiv bestimmten der Schule Karl Büchers in Leipzig ziemlich deutlich abheben kann, ist die aus verschiedenen Quellen gespeiste idealistische Frage nach der Leistung (und darïber hinaus: Leistungsfähigkeit, Wirkmöglichkeit) des Publizisten als des Gestalters und Führers der „Offentlichen Meinung“. Das Publikum wurde mitgedacht als zu führende und möglichst auch zu belehrende (d. h. in ihrem Gesamtniveau zu bebende) Empfängerschaft. Obwohl man sich von der dem 19. Jahrhundert angehörigen Vorstellung einer einhelligen Offentlichen Meinung bereits kritisch freigemacht hatte ${ }^{3}$, fïhrte man die Entmythologisierung dieses Begriffes nicht konsequent zu Ende. Selbst bei Hagemann, der die Aussage zum wichtigsten Fragegegenstand erklärt hatte, blieb der Publizist Schlüsselfigur, die mit Hilfe von Methoden und Techniken, ausgestattet mit einem spezifischen publizistischen Ethos, grundsätzlich, wenn auch durch mancherlei Bedingungen eingeschränkt, erheblichen Einfluß auf die Empfänger ausüben kann. Die in den 20er und 30er Jahren enge Verbindung der publizistikwissenschaftlichen Lehre mit der journalistischen Praxis, bei der die Lehre übrigens wenig Gegenliebe fand, führte ebenfalls dazu, daß man dem praktischen Publizisten möglichst umfassende Handlungsregeln auf den Weg geben wollte, die in ihren werthaltigen Kategorien die Wertungen der Lehrenden widerspiegelten. Die von Dovifat immer wieder betonten "Gesinnungskräfte" ${ }^{{ }_{4}}$ als movens aller Publizistik sollten natürlich im „Normal-

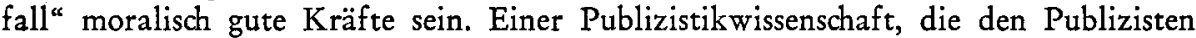
als Volksführer oder -lehrer sah, war wertende Beurteilung publizistischer Sachverhalte so selbstverständlich, daß es ihr gar nicht oder nur in Einzelfällen einiger 
Spezialuntersuchungen in den Sinn kam, daß diese Wissenschaft auch nicht-wertend betrieben werden könnte. (Die Tatsache, daß katholische Gelehrte die traditionellkirchliche Unterscheidung zwischen "guter" und "schlechter" Presse gewissermaßen von klein auf hatten verarbeiten müssen, mag hierbei auch eine Rolle spielen.) Die erst geraume Zeit nach dem Zweiten Weltkrieg wieder erhobene Forderung nach wertfreiem Vorgehen auch in der Publizistikwissenschaft - ähnlich wie in den neueren Richtungen der Sozialwissenschaften - führte zu Auseinandersetzungen $z$ wischen prominenten Fachvertretern ${ }^{5}$ und gelegentlich zur Bezeichnung der älteren Publizistik als normative Wissenschaft, was sie im eigentlichen Sinne nie war. Daß man Methoden neutral anzuwenden habe, darüber besteht zwischen den Vertretern der alten und der neueren „Philosophie" vermutlich größere Ubereinstimmung, als man ihren Außerungen entnehmen kann. Der Unterschied liegt im Problen der Interpretation wie immer gefundener Forschungsergebnisse und in der Frage, ob man publizistische Sachverhalte aufgrund wissenschaftlicher Einsicht und Erfahrung wertend beurteilen oder nur analysieren darf.

Wesentlich gekennzeichnet werden jedoch die neuen Ansätze des Faches durch die beiden folgenden Forderungen:

1. die Forderung nach einer (am Ende) allgemeinen Theorie der publizistischen (d. h. öffentlichen und aktuellen) Kommunikation,

2. die Forderung nach stärkerer Einbeziehung der Publikumserforschung, weil nur auf diesem Wege die Ganzheit des Kommunikationsprozesses erfaßt werden kann.

Der hier verwendete Theoriebegriff ist nicht deckungsgleich mit dem naturwissenschaftlichen, aber doch weit entfernt von der in den letzten Jahrzehnten in den Geisteswissenschaften meist sehr unbestimmten Verwendung des Begriffes Theorie. Ein eher naturwissenschaftliches Vorbild für die Begriffsausformung wird man, betrachtet man gleichzeitig die methodologische und erkenntnistheoretische Entwicklung dieser Richtung ${ }^{6}$, nicht leugnen können. Wertneutralität ist, will man zur Formulierung (nahezu gesetzmäßiger) Regeln möglichst weiten Geltungsbereichs vordringen, Vorbedingung, mindestens als wissenschaftsmoralisches Prinzip ${ }^{7}$.

$\mathrm{Da}$ die jüngere Publizistikwissenschaft alle bisher auf dem Wege zur Theorie unternommenen Schritte wohltuend selbstkritisch beurteilt, spricht man vorerst nur von Ansätzen und lediglich im optimistischen Falle von der Hoffnung, dicht vor der Bildung einer Theorie $\mathrm{zu}$ stehen $^{8}$.

Als ein solcher Ansatz ist das zu verstehen, was der niederländische, in Münster lehrende Publizistikwissenschaftler Henk Prakke unter dem Markenzeichen „Funktionale Publizistik" in die Fachdiskussion eingebracht hat. In einigen selbständigen Schriften und knappen Aufsätzen ${ }^{9}$ hat er seine Vorstellungen vom publizistischen Kommunikationsprozeß entwickelt und sie schließlich zusammen mit seinen Mitarbeitern in Münster in dem Buch "Kommunikation der Gesellschaft"10 systematisiert und konzentriert. Auch diese Vorarbeiten zu einer Theorie gehen von einer forcierten Einbeziehung des Publikums in die Betrachtung des publizistischen Prozesses aus, der grundsätzlich als (hypothetisch gesetzmäßiges) stets interdependentes Wechselspiel zwischen Publizist und Publikum (Kommunikator und Rezipient) gesehen wird. Ohne daß wir auf Einzelheiten dieser Vorstellung eingehen, heben wir hervor, daß in diesem - in seinen wechselseitigen Abhängigkeiten einer mathematischen Funktion ähnelnden - Kommunikationsverhältnis die Rolle des Rezipienten nicht nur gleichgewichtig zu der des Kommunikators gesehen wird, sondern daß sie auch nicht "wegdenkbar“ ist: Kommunikator oder Aussage oder Medium 
können, wenn man Allgemeines über den Prozeß erkennen will, nicht mehr separiert untersucht werden. Kommunikator und Rezipient zusammen effektuieren, was und wie in der öffentlichen Kommunikation zur Mit-Teilung gebracht wird. Die Bedürfnisse des Publikums, deren Präsenz bei den Kommunikatoren als Inspiration aktiv wird, steuern Inhalt, Umfang und Gestaltung des publizistischen Angebots, ein Rückkopplungsvorgang, der Prakkes Schüler und Mitautor Franz W. Dröge zur Bezeichnung der Publizistik als "Regelungslehre" geführt hat ${ }^{11}$. Für den Publizisten als Führer oder gar Verführer ist in ihr wenig Platz, es sei denn, daß die Bedürfnisse des Publikums auf den Führer oder Verführer aus sind. In die platte Redewendung ïbertagen heißt das: Jede Gesellschaft hat die Publizistik, die sie verdient. Oder in Beispielen gesprochen: Eine Gesellschaft von Heiligen oder wissend Heilsbedürftigen könnte durch ihre den Publizisten inspirierenden Bedürfnisse bewirken, daß die Zehn Gebote Thema der aktuellen Kommunikation werden, d. h. in der journalistischen Praxis: Schlagzeilen abgeben, Titelseiten prägen. Eine Gesellschaft von Unvollkommenen inspiriert die Kommunikatoren eher dazu, Titelseiten immer häufiger mit Aktfotos zu füllen, und der wachsende Absatz so gestalteter Blätter bestätigt ihren Machern, daß sie die von ihrem Publikum ausgegangene Inspiration richtig umgesetzt haben. Beide Möglichkeiten sind in der funktionalistischen Vorstellung von Publizistik vorgesehen. Die Geschichte der Publizistik lehrt, daß das Publikum meist zur Verwirklichung der zweiten beizutragen geneigt ist. Die Tatsache, daß der publizistische Erfolg (auch jener, der sich auszahlt) seit eh und je bei dem angenebmste Bedürfnisbefriedigung versprechenden Angebot lag, ist von Kritikern, die in den publizistischen Medien eher Einrichtungen zur Hebung und Bildung des Volkes als gesellschaftsstabilisierende Kommunikationsinstrumente sahen, of genug angegriffen worden. Als der Generalanzeiger längst als erfolgreicher, aber noch nicht überall als zweckmäßig nachrichtenvermittelnder Zeitungstyp anerkannt war, hieß es: „Der Generalanzeiger sucht nicht Einfluß auf die Leserschaft zu gewinnen, sondern gerade im Gegenteil, sich von der Leserschaft beeinflussen zu lassen. " 12

Damit ist der Sachverhalt genau beschrieben, zugleich aber die Gefahr, wie die funktionalistische Sicht der Publizistik auch verstanden werden kann. Ob sie tatsächlich so, als die Theorie zur Rechtfertigung der angenebmen Bedarfdeckungspublizistik, mißverstanden werden darf, wird sie selbst durch eigene Untersuchungen zu klären haben. Prakkes These, daß im "Produkt der Begegnungen“, der Kommunikation, beide Seiten, Kommunikator wie Rezipient „in jeder beliebigen Zusam-

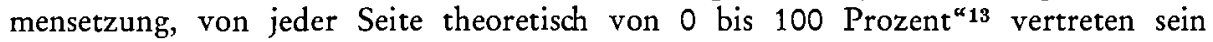
könnten, wird durch folgende zwar tendenziöse, aber dennoch genaue Schilderung aus längst vergangener Zeit angefochten: „Drum ist erstes Prinzip der Zeitungsindustrie, Abonnenten heranzuziehen, denn diese sind zunächst die Hauptsache. Sie kommen aber nur, wenn ihnen die Zeitung zusagt. Der Preßgeschäftsmann sucht darum zu erforschen, welche Absicht dem großen Publikum die angenehmste ist, was dasselbe am liebsten hört oder liest. Manche haben es in dieser Beziehung zu einer erstaunlichen Feinfühligkeit gebracht. Hat man erst heraus, was den meisten Abonnenten genehm ist, so ist das Weitere verhältnismäßig leicht... Man fragt nicht sonderlich darnach, ob das, was in die Zeitung kommt, wahr oder falsch, richtig oder unrichtig, Tatsache oder Erfindung, gut oder böse ist, sondern ob es die Abonnenten befriedigt und geeignet ist, neue Abnehmer heranzulocken. Auf einen bestimmten Parteistandpunkt stellt man sich so lange, als das Publikum dadurch angezogen wird, um ihn alsbald zu verlassen, wenn durch Vertretung 
anderer Anschauungen mehr Abonnenten zu gewinnen sind. Nützt es der Verbreitung des Blattes, so redet man besonnen, ehrbar, verständig, mit sittlichem Pathos, und wiederum ist man keck, unbesonnen, frivol, man spottet, höhnt, verleumdet, lügt, bringt sensationelle Nachrichten, pikante Erzählungen, spricht ohne Scheu dem Ehrbaren Hohn, wenn sich dadurch die Zahl der Abnehmer mehrt. "14

Ubersetzte man diesen Passus in die Sprache der Publizistikwissenschaft, so erhielte man eine Parodie auf die funktionalistische Deutung des Verhältnisses zwischen Publizist und Publikum. Prakke würde solch ein Vorgehen Kulturpessimismus nennen. ${ }^{15}$ Er kann sich darauf berufen, daß in der "Kommunikation der Gesellschaft" als der jüngsten Darstellung des interdependent funktionierenden Kommunikationsverhältnisses dem Publizisten immerhin ein gewisser Freiheitsspielraum für seine Wirkmöglichkeit zuerkannt ist. Wo die Grundleistungen aller Publizistik als Spiegelung und Prägung beschrieben werden, wird für die Spiegelung von vornherein Übereinstimmung von Publikumserwartung und publizistischem Angebot angenommen. Für die Prägung jedoch gilt, daß der Kommunikator etwas mehr oder jedenfalls anderes anbietet als nur das, was von ihm erwartet wird.16 Akzeptiert der Rezipient dieses "Mehr ${ }^{\circ}$, das man als Differenzbetrag zwischen Erwartung und Angebot bezeichnen kann, so beugt er sich (zunächst einmal im bloßen Annehmen) dem Kommunikator. Alle intentional einsetzbaren Führungs-, Beeinflussungs-, Bildungs- oder auch Verdummungsqualitäten, die man der Publizistik nach wie vor gern zuschreibt, sind in dieser Differenz-Spanne beschlossen. Wird sie vom Publizisten überspannt, so verweigert das Publikum die Annahme, - Kommunikation kommt nicht zustande, und Prägung bleibt ganz und gar aus. Als Beispiel seien die offenbar recht gefestigten Erwartungen erwähnt, die das Publikum gegenüber Illustrierten-Titelbildern hegt: „Als der Stern die Serie der weiblichen Starporträts durchbrach und es riskierte, Adenauer auf der Titelseite abzubilden..., ging die Auflage dieser Ausgabe um 60000 zurück: Adenauer brachte Verluste. "17 Selbst „Mann in der Zeit" und "Gong ${ }^{\text {“ }}$ beugen sich in letzter Zeit dem gebieterischen Erwartungsdruck und bringen weibliche Porträts auf der Titelseite.

Welchen Umfang die für publizistische Prägung verfügbare Spanne unter je verschiedenen historischen Umständen annimmt, ist noch eine Forschungsaufgabe für die Wissenschaft. Spektakuläre Fälle wie etwa die Gleichschaltung der Publizistik unter dem nationalsozialistischen Regime können zwar zu der Annahme verleiten, daß man mit der Publizistik "alles machen“ könne, aber sie sagen nichts darüber, ob unter solch einseitig überspannten Umständen auch wirklich Kommunikation stattfindet. Anders gefragt: Wieviele Leser der mit hohen Auflagen ausgestatteten Blätter von der Art des „Völkischen Beobachter“ mögen ihre Zeitung nur als pflichtmäßiges Feigenblatt, aber nicht als Kommunikationspartner akzeptiert haben? Wieviele Gebührenzahler totalitärer Rundfunkanstalten richten ihre Antennen auf Sender, von denen sie sich mehr Information oder angenehmere Unterhaltung erwarten? Aus den bisherigen Beobachtungen kann man sagen, daß die für Prägung zur Verfügung stehende Differenz-Spanne in den meisten Fällen eher klein als groß sein dürfte. In sie muß platterdings alles "Höhere* hineingepackt werden, was der kritische Bürger idealistischer Bildungstradition der Publizistik als Kulturaufgabe zuweist. (Oft genug, ohne es für sich selbst als Leistung wirklich zu erwarten.) Der funktionalistische Theorie-Ansatz muß nun aber so interpretiert werden, daß das "Höhere“, also etwa Bildung, Moral, ausführliche Information, unter Umständen Doktrin oder auch Ideologie - kurzum: Werte und "Gesinnungskräfte" - nur in homöopathischen Dosen in den Kommunikationsfluß eingebracht werden darf, 
soll das Kommunikationssystem nicht zusammenbrechen. Alle Kommunikatoren oder Kommunikationseinrichtungen, die auch oder in erster Linie wirtschaftlich am Funktionieren der öffentlichen Kommunikation interessiert sind, also z. B. Verleger, kommerzielle Rundfunkanstalten, werden den Zusammenbruch des Kommunikationssystems naturgemäß scheuen; er bedeutet für sie sinkende Auflagen, schwindende Teilnehmerzahlen, wirtschaftliche Verluste. Sie werden also mit der prägenden Uberstrapazierung der Publikumserwartungen außerordentlich vorsichtig verfahren und möglicherweise, man betrachte etwa unsere Wochenendpresse, völlig darauf verzichten; d. h. sie werden sich ganz den Publikumserwartungen anpassen. Im Sinne der funktionalistischen Sicht des Kommunikationsprozesses ist dies weder gut noch böse, sondern eigentlich sachgerecht. Gekonnte Anpassung dient der Stabilisierung der für das Bestehen der modernen Industriegesellschaft notwendigen Kommunikationssysteme. Zugleich aber stellt sich die Frage nach einer Grundlegung der publizistischen Moral, die sich nicht funktional beschreiben läßt; denn ganz offenbar ist die Moral des Publikums in vielen Fällen mit Nicht-Wahrheiten (unvollständige Information, erfundene Ereignisse, aufgcbauschte Nichtigkeiten), gelegentlich sogar mit Lügen zufrieden. Der Kommunikator wird durch so "niedrig“ nivellierte Erwartungen dazu inspiriert, es sich leicht zu machen. Falls ihm dies aus moralischen oder berufsethischen Erwägungen schwer fällt, bleibt ihm oft nicht einmal das Wort an sein Publikum, weil er damit möglicherweise die freie Spanne der Prägung überschritte und also gar nicht gehört würde, sondern nur der Rückzug, etwa durch Austritt aus einer Redaktion. Unmittelbar im Gefolge einer funktionalen Publizistik tritt also, paradoxerweise stärker als für die Gedankengänge der wertenden Publizistik, das Desiderat einer publizistischen Ethik auf, die, wohlgemerkt, etwas anderes zu sein hätte als eine Sammlung von Forderungen der allgemeinen Moral an die Publizistik. Vielmehr hätte sie die Eigengesetzlichkeit der Publizistik, wenn sie erst einmal zutreffend erkannt sein sollte, in ihr Koordinatensystem einzubeziehen.

Der meßbar erfolgreiche Kommunikator hätte nach dem beutigen Stand der Dinge (im Unterschied zu den erfolglosen Produzenten von „Papas Kino") das auch wissenschaftlich $\mathrm{zu}$ sichernde Argument für sich: Das Publikum will es so. Die gern und leicht geübte Kritik an den bestehenden publizistischen Verhältnissen müßte sich mindestens teilweise von den publizistischen Organen ab- und dem Publikum zuwenden. Eine Gesellschaft, die auf moralische Vervollkommnung abzielte, würde - wenn die funktionalistische Sicht richtig ist - eine moralisch einwandfreie Publizistik hervorbringen, ein bildungsbeflissenes Publikum könnte den Goldenen Schuß verschwinden und das Studienfernsehen Triumphe feiern lassen, wie im historischen Beispiel in der Tat eine in maßgeblichen Schichten imperialistisch interessierte Gesellschaft eine ganze Reihe imperialistisch indoktrinierender Organe hervorgebracht hat, für die in unserer heutigen Gesellschaft kaum eine Erwartungslage bestünde.

Der funktionalpublizistische Theorieansatz setzt nun fürwahr eher Optimismus als Kulturpessimismus voraus. Wer an den durch Aufklärung zu befördernden Fortschritt der Menschheit glaubt, sieht die Verbesserung der kommunikativen Verhältnisse beinahe automatisch mit heraufziehen. Auch ein demokratisches Ingrediens ist nicht $\mathrm{zu}$ übersehen. Funktionalistisch gesehen ist der (ausgerechnet von den überzeugten Anhängern des Aufklärungs-Fortschrittsprinzips als Machtkonzentration gefürchtete) zahlenmäßige Erfolg etwa der „Bild-Zeitung“ der funktionierenden Adäquanz zwischen Angebot und Erwartungen zuzuschreiben: Der tägliche Verkauf 
von mehr als vier Millionen ist tägliche Abstimmung und liefert je frische empirische Daten zur Kontrolle des Funktionierens.

Wer die Geschichte der Menschheit eher skeptisch zu betrachten geneigt ist, dem fehlt im funktionalistischen Modell, - und das mag an dessen philosophischen Fundamenten liegen, - die Einbruchsstelle des Außerordentlichen. Man kann sagen: Der ungewöhnliche Missionserfolg eines Paulus kam auf Grund der religiösen Bedürfnislage seiner mittelmeerländischen Zeitgenossen zustande. Oder man kann sagen: Der Erfolg der Kreuzzugspredigten Bernhard von Clairvaux' war mindestens zur Hälfte auf die vorgegebene allgemeine Kreuzzugsbereitschaft zurückzuführen. Aber man sollte auch den Gründen nachgehen, die Joseph Lortz zu der Erklärung veranlaßten: „... seine ganze Gestalt predigte und wurde zum unauslöschlichen, hinreißenden Eindruck. Sein Geheimnis war die Fähigkeit, andere mit sich zu reißen, sei es durch die von ihm ausströmende, wogende Begeisterung, sei es durch eine erschreckend übermenschliche Befehlsgewalt, wie seine Lebensbeschreibung berichtet." 18 Wenn nun die „publizistische Persönlichkeit“, die als Typus die ältere Publizistikwissenschaft so faszinierte, doch nicht pur funktionalistisch erklärbar wäre? Wenn die im Bild des welt-immanenten Regelkreises endende funktionale Publizistik im wissenschaftlich veranlaßten Vertrauen auf die menschlich-fortschrittliche Selbstregulierung und also die Machbarkeit der Dinge endete? Wenn darüber wichtige Fragen nach dem Problem des in der gesellschaftlichen Entwicklung vielleicht weiterführenden Ausbruchs aus dem publizistischen Regelkreis unterblieben? Das sind Fragen, die man nicht an den Anreger der funktionalen Sicht der Publizistik richten darf. Gerade Prakke hat nämlich neben der funktionalen Sicht auch die „Erfahrung des Heiligen " 19 als Gegenstand in das Fachgespräch neu eingebracht. Es sind vielmehr Fragen, die zur Beweisführung für einen Theorie-Ansatz herausfordern. Zwar vermag er schon jetzt vieles, was die Kritiker seit 200 Jahren und heute besonders wieder an der Publizistik aufregt, erheblich ruhiger'mit dem Hinweis auf eine zu beachtende Eigengesetzlichkeit $\mathrm{zu}$ erklären. Einem Publikum, das sich nach wie vor der Macht der Offentlichen Meinung oder neuerdings der Massenmedien ausgeliefert fühlt, wird er sich allmählich überzeugend verständlich machen müssen. Vorläufig können wir es schon als einen Gewinn verbuchen, wenn eine neue, sachliche, im Hinblick auf die Selbstregelung der Angebots- und Erwartungsstrukturen vielleicht $\mathrm{zu}$ optimistische Sicht publizistischer Phänomene auch zu einem neuen Verstehen alter Weisheiten führt. 1848, in einem Jahr hochgeschraubter Publikumserwartungen, schrieb das münstersche "Sonntagsblatt für katholische Christen": „Die weltliche Behörde hat auf ihr Aufsichtsrecht über die Presse zu Gunsten des lesenden Publikums, welches dasselbe für sich in Anspruch genommen, verzichtet. Jedem Rechte aber steht eine Pflicht zur Seite... die Pflicht, sich den verderblichen Einwirkungen der Presse in jeder Hinsicht zu verschließen und sich mit umso größerer Sorgfalt dagegen zu verwahren. "20 Pflicht für das um 120 Jahre erfahrenere Publikum ist nicht mehr, sich zu verschließen, sondern die eigene Position und Funktion im Kommunikationsprozeß zu erkennen und sich entsprechend $\mathrm{zu}$ verhalten. Das gilt, spätestens seit dem II. Vaticanum, auch „für katholische Christen“, wie sie weiland das Sonntagsblatt ermahnte.

Anmerkungen:

1. Karl Bömer: Bibliographisches Handbuch der Zeitungswissenschaft, Leipzig 1929, und Internationale Bibliographie des Zeitungswesens, Leipzig 1932.

2. Josef H. Krumbach: Vorfragen einer Soziologie der Katholischen Presse, München 1932; Grundfragen der Publizistik, Berlin und Leipzig 1935, u. a. m. 
3. Vgl. etwa Walter Hagemann: Grundzüge der Publizistik, Münster \&1966, S. 55 f.

4. Vgl. etwa Emil Dovifat: Zeitungslehre Bd. I, Berlin ${ }^{3} 1955$, S. 22, ${ }^{5} 1967$, S. 6 und neuerdings in seinem Handbuch der Publizistik Bd. I (Allgemeine Publizistik), Berlin 1968, S. 5 und S. 29-34 (Abschnitt "Gesinnung": „Alle Publizistik ist gesinnungsbestimmt." S. 34.)

5. Vgl. Fritz Eberhard: Thesen zur Publizistikwissenschaft, in: „Publizistik“, Bremen, 6. Jg. 1961, S. 259-266, und Emil Dovifat: Ergebnisse der Publizistikwissenschaft, in: „Publizistik“, 7. Jg. 1962, S. 78-81.

6. Vgl. etwa: Elisabeth Noelle-Neumann: Die Rolle des Experiments in der Publizistikwissenschaft, in: „Publizistik“, 10. Jg. 1965, S. 239-250; Gernot Wersig: Zum Problem der wissenschaftstheoretischen Einordnung der Publizistikwissenschaft, in: Zur Lage von Publizistik- und Zeitungswissenschaft in der Bundesrepublik Deutschland und West-Berlin, Berlin, Ausgabe 1965, S. $11-20$, bes. S. 17 f.; als Überblick: Gerhard Maletzke: Publizistikwissenschaft zwischen Geistes- und Sozialwissenschaften, Berlin 1967.

7. Uber die Möglichkeit korrekter Einhaltung von "Wertneutralität“ oder gar Wertfreiheit kann man selbst bei rein quantifizierenden Untersuchungen streiten, sobald menschliches Handeln oder Sich-Verhalten Untersuchungsgegenstand ist. Sowohl in die Fragestellung als auch in die Ergebnisdarstellung mischen sich notwendig subjektivinterpretative Komponenten.

8. So mit dem Amerikaner Herbert H. Hyman Elisabeth Noelle-Neumann in ihrem Aufsatz über Meinung und Meinungsführer in: „Publizistik“, 8. Jg. 1963, S. 316-323, hier S. $320 \mathrm{f}$. Kritisch zu Hyman: Franz W. Dröge und Winfried B. Lerg: Kritik der Kommunikationswissenschaft, in: Festschrift für Otto Groth, Bremen 1965, S. 67-100, hier S. 95.

9. H. J. Prakke: Van perswetenschap to publicistiek, Assen 1956, -- De samenspraak in onze samenleving, Assen 1957, - Alle Publizistik ist Zwiegespräch, in: „Publizistik“, 5. Jg. 1960, S. 208-210, - Uber die Entgrenzung der Publizistik, Assen 1961, - Thesen zu einer neuen Definition der Publizistikwissenschaft, in: "Publizistik“, 6. Jg. 1961, S. $81-84$.

10. Henk Prakke, Franz W. Dröge, Winfried B. Lerg, Michael Schmolke: Kommunikation der Gesellschaft. Einführung in die funktionale Publizistik, Münster 1968.

11. Franz W. Dröge: Regel und Regelung. Ansätze der Publizistikwissenschaft, in: „Publizistik“, 11. Jg. 1966, S. 145-152, hier S. 149.

12. Karl Wies: Brennende Fragen der katholischen Presse, Trier 1930, S. 6.

13. Prakke: Alle Publizistik ist Zwiegespräch, a. a. O. S. 210.

14. Johannes Frizenschaf zitiert hier den anonymen Autor einer 1880 in Barmen veröffentlichten Schrift „Wic können die Schäden unserer periodischen Presse dauernd geheilt werden?" Frizenschaf: Die Wahrheit über die farblose Presse, Bochum o. J. (1912), S. 15. Von der gleichen moralischen Grundfeste geht übrigens neuerdings Hans Dieter Müller in seiner "kritischen Studie“ "Der Springer-Konzern“ (München 1968) aus, wenn er etwa (S. 11) nicht "glaubt“ (wie er es formuliert), „daß Erfolg [hier der Erfolg des Springer-Konzerns] schon der Beweis für die Richtigkeit eines Phänomens ist". Auch die Schlußsätze aus Müllers Buch (S. 310) sind in unserem Zusammenhang interessant: Axel Springer habe, am Ende den geschäftlichen Erfolg für etwas Höheres" genommen, - „für Wink, Weisung, Bestätigung seiner Ideen“... "das ,Volk' stimme quasi täglich demokratisch für ihn ab, habe ihm die politischpublizistische Macht als, Lehen' verliehen. Darin mag, hintergründig und unfreiwillig, ein Stück Wahrheit über die wahre Verfassung unserer Gesellschaft liegen: die vollendete Anpassung an die Konsumbedürfnisse, die Perfektion des Verkaufsjournalismus als politisches Mandat."

15. Prakke: Alle Publizistik ist Zwiegespräch, a. a. O. S. 209.

16. Prakke e. a.: Kommunikation der Gesellschaft, a. a. O. S. 92-95.

17. Hans Jürgen Usko, Günter Schlichting: Kampf am Kiosk. Macht und Ohnmacht der deutschen Illustrierten, Hamburg 1961, S. 16.

18. Joseph Lortz: Geschichte der Kirche in ideengeschichtlicher Betrachtung, Münster 201959, S. 160. 
19. Henk Prakke im Vorwort zu Franz-Josef Eilers: Christliche Publizistik in Afrika, Steyl 1964, S. 8.

20. "Sonntagsblatt für katholische Christen“, Münster, 7. Jg. 1848 (Nr. 13, 26. März), S. 217--224. Hier zit. nach Rudolf Pesch: Die kirchlich-politische Presse in der Rheinprovinz, Mainz 1966, S. 314.

\section{S UM M A R Y}

The old German "Zeitungswissenschaft" (Science of Journalism) is a thing of the past. One new direction toward an overall research in the processes of public communication is in its self-explanatory term the "Funktionale Publizistik " (Functional Communication Research), an approach inaugurated by Professor Henk Prakke at the University of Münster. In this effort to discover a theory of mass communications the exchange of messages between the communicator and the recipient is regarded as a relation of interdependence. Changing the variable "communicator" means also changing the variable "recipient" and vice-versa. The explanation of this process resembles a feedback control system. Some have the impression that the communicator is forced to adaptation by the recipient. The idea that mass communication - on account of the communicator's intentions - immediately must lead or is capable of leading the public to a higher degree of education and human evolution cannot be accepted any longer. Where in the "Funktionale Publizistik" approach is there any room left for the "higher level“ and moral control? Apparently all this is strictly limited. Will the result be a communication system leading to adaptation? How would the communicator (the journalist) react when he feels himself limited by the moral code of his profession? The functionalistic view of mass communications is able to explain many a thing more convincingly than former attempts. On the other hand, it seems that the ethical question in mass communications is still lacking, namely, that practice must agree with theory. The explanation with the metaphore of a "feedback control system" does not satisfy every detail, because it can consider the invasion of extraordinary phenomena of mass communications into a feedback control system only insofar as this invasion causes the break-down of a communication system.

\section{RESUMEN}

La antigua ciencia del periodismo alemana ("Zeitungswissenschaft") pertenece al pasado. Una de las nuevas corrientes, que se esfuerza en investigar el público proceso de comunicación, es la que se llama a sí misma "periodismo funcional“, y que tiene su origen en el enseñante de Münster, Profesor Henk Prakke. Considera al intercambio fundamental de comunicación entre el comunicador y el receptor, como una relación de interdependencia. Las mutaciones del comunicador cambian también al receptor y viceversa. Es fácil explicar este proceso como un círculo regular y ya se ha realizado. Se puede tener la impresión de que, en este proceso, el recipiente o receptor obliga al comunicador a una acomodación. Así, pues, la idea de que el periodismo debe y puede llevar directamente al público a una mayor educación y desarrollo humano, a causa de las intenciones de los comunicadores, no puede continuar defendiéndose de esta manera. ¿ Dónde existe lugar en el periodismo funcional para influjos de „más alto nivel“ y dirección moral? Evidentemente, el está muy limitado. Es una consecuencia el periodismo acomodatício? ¿ Cómo se comporta el comunicador (periodista) consciente del código moral de su vocación? La visión funcional del periodismo explica algunas cosàs de manera más convincente que otras tentativas anteriores. Pero también ella presenta una respuesta que deja mucho que desear a la pregunta sobre una ética del periodismo que concuerde con la teoría. La explicación con ayuda del circulo regular arriba mencionado no satisface plenamente, porque solo puede prever la invasión de fenómenos extraordinarios del periodismo en el círculo regular de tal manera que, a través de ella, se realice el derrumbamiento de un sistema de comunicación. 